\title{
Choque cardiogénico secundario a infarto agudo al miocardio con cetoacidosis diabética grave: reporte de un caso
}

\author{
Cardiogenic shock secondary to acute myocardial infarction with severe diabetic \\ ketoacidosis: a case report \\ Alejandro Canedo-Alberto*, Francisco J. Franco-Núñez y Carmen A. Bartolón-Gómez \\ Servicio de Urgencias, Hospital General Regional No. 1, Instituto Mexicano del Seguro Social (IMSS), Morelia, Michoacán, México
}

\section{Introducción}

Las enfermedades cardiovasculares son la principal causa de muerte en el mundo, representando más del $30 \%$, principalmente el infarto agudo al miocardio (IAM) con elevación del segmento ST (IAMCEST) y sus complicaciones. En México, el Instituto Nacional de Estadística y Geografía, en el año 2013, registró 116,002 defunciones por cardiopatía isquémica'.

La última revisión de la cuarta definición universal de infarto al miocardio define la lesión miocárdica como la presencia de un aumento o una disminución de los valores de troponinas cardiacas al menos un valor por encima del percentil 99. EI IAM debe presentar síntomas de isquemia miocárdica, nuevos cambios isquémicos en el electrocardiograma (ECG), desarrollo de ondas $Q$ patológicas o evidencia por imagen ecocardiográfica².

El proceso fisiopatológico en común de los síndromes isquémicos coronarios agudos es la oclusión del flujo coronario, principalmente por ruptura de una placa vulnerable ${ }^{3,4}$.

La presentación clínica del IAM tiene como síntoma principal el dolor torácico opresivo y disneizante, acompañado por síntomas adrenérgicos. El ECG debe realizarse en los primeros 10 minutos del contacto con el paciente. Existen presentaciones atípicas del dolor, con mayor prevalencia en ancianos y diabéticos. Los equivalentes anginosos pueden presentarse como edema agudo pulmonar, crisis hipertensivas, disnea, flutter paroxístico o fibrilación auricular, disnea súbita, arritmias cardiacas, bloqueo auriculoventricular e isquemia en el ECG ${ }^{5,6}$.

El objetivo del tratamiento del IAMCEST es la reperfusión coronaria precoz. Si se dispone de una sala de hemodinamia debe realizarse una intervención coronaria percutánea; si no se tiene el recurso, se realiza reperfusión coronaria farmacológica, además del tratamiento antiisquémico ${ }^{7}$.

Las complicaciones del IAMCEST pueden ser mecánicas o eléctricas (bradiarritmias y bloqueos, arritmias ventriculares). La prevalencia del choque cardiogénico varía del $6 \%$ al $15 \%$, y se presenta sobre todo en los pacientes con complicaciones mecánicas; representa la principal causa de muerte en pacientes hospitalizados con IAM, oscilando entre el $40 \%$ y el $60 \%$. Es importante comentar que la cetoacidosis diabética (CAD) puede presentarse en conjunto con el IAM, y para esta combinación la mortalidad ronda el 85\%

\section{Presentación del caso}

Varón de 53 años, originario de Nueva Italia, residente de Morelia, Michoacán, campesino, con primaria incompleta, casado y católico.

\section{Correspondencia:}

*Alejandro Canedo-Alberto

E-mail: medicuscanedo@outlook.com
Fecha de recepción: 29-11-2021 Fecha de aceptación: 21-12-2021 DOI: 10.24875/REIE.21000207
Disponible en internet: 07-03-2022
Rev Educ Investig Emer. 2022;4(Supl 1):90-94
www.medicinadeemergencias.com www.medicinadeemergencias.com bajo la licencia CC BY-NC-ND (http://creativecommons.org/licenses/by-nc-nd/4.0/). 
Traído por familiares al servicio de urgencias por sus medios, procedente de su área laboral por presentar síncope en dos ocasiones, el primero a las 12:00 h, con recuperación parcial del estado de despierto, presentando debilidad, diaforesis y somnolencia, por lo que es retirado de área laboral; posteriormente, a las 16:00 h presenta un nuevo evento de síncope sin recuperación del estado de despierto, indiferencia al medio y con nula respuesta a estímulo verbal o táctil.

Entre sus antecedentes se encuentran carga genética por ambas ramas para hipertensión arterial y diabetes mellitus. Diagnosticado de diabetes tipo 2 hace 15 años, en tratamiento a base de biguanidas y sulfonilureas con mal apego. Amputación quirúrgica del tercer dedo del miembro pélvico izquierdo hace 15 años. Sedentario. Alcoholismo social cada 1 a 2 meses, sin llegar a la embriaguez. Niega tabaquismo.

Como factores de riesgo cardiovascular podemos identificar el sexo masculino, el sedentarismo y la diabetes mellitus.

Ingresa a sala de reanimación por hipotensión (80/40 mmHg), bradicardia (30 latidos por minuto) e hiperglucemia $(501 \mathrm{mg} / \mathrm{dl})$ en triaje. El paciente se encuentra en malas condiciones generales, con franco deterioro neurológico, soporoso, con indiferencia al medio, nula respuesta a estímulo verbal o álgido, mucosas pálidas y subhidratadas, diaforético, con palidez tegumentaria generalizada, pupilas anisocóricas, izquierda de $2 \mathrm{~mm}$ y derecha $3 \mathrm{~mm}$, reflejo consensual y fotomotor disminuidos, pero presentes. Tórax normolíneo, con ruidos respiratorios anormales, polipneico, con mala mecánica ventilatoria, estertores finos inspiratorios en la región paraesternal bilateral, no sibilancias. Precordio arrítmico, bradicardia de 30 latidos por minuto, intensidad disminuida, no se ausculta S3 ni S4. Abdomen blando, no doloroso, sin datos de irritación peritoneal. Extremidad inferior izquierda con ausencia del tercer dedo del pie, llenado capilar de 5 segundos, presencia de mottling en ambas piernas, hasta la región inguinal, pulsos distales presentes, pero disminuidos en intensidad y frecuencia, no edema, Babinski negativo.

\section{Abordaje}

A su ingreso a la sala de choque se realiza monitorización continua de los signos vitales, identificando bradicardia e hipotensión, además de saturaciones bajas (80-82\%) al aire ambiente. Se procede a realizar

ECG en los primeros 10 minutos tras su ingreso, identificando IAMCEST en cara inferior y datos de bloqueo auriculoventricular completo, además de elevación del

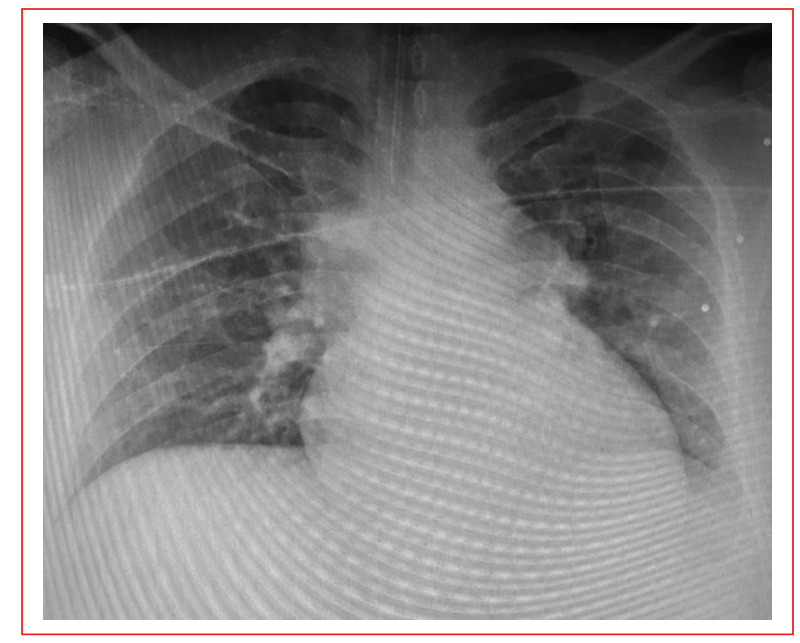

Figura 1. Radiografía de tórax al ingreso.

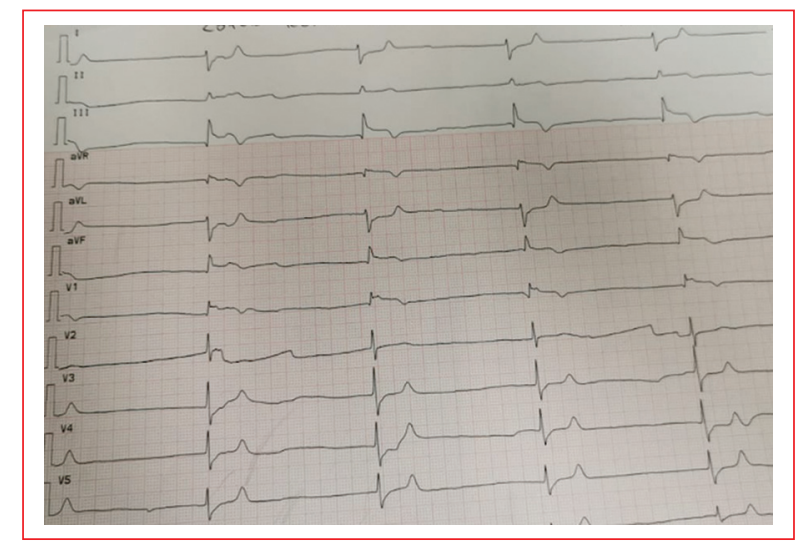

Figura 2. Electrocardiograma de ingreso al servicio de urgencias.

segmento ST en derivaciones derechas V3R-V6R. Dadas las condiciones clínicas del paciente, se realiza soporte vital básico y avanzado, se canulan dos vías periféricas de calibre $16 \mathrm{Fr}$, se obtienen muestras de laboratorio y se realiza manejo avanzado de la vía aérea con la secuencia de inducción fármaco-asistida La figura 2 muestra el electrocardiograma de ingreso del paciente. Durante su monitorización se corroboró bradicardia secundaria a bloqueo auriculoventricular completo, teniendo como probable etiología la cardiopatía isquémica. La figura 1 muestra la radiografía de tórax posterior a intubación.

\section{Diagnósticos diferenciales}

- Síndrome coronario agudo.

- Diabetes mellitus tipo 2 descompensada. 
Tabla 1. Orden cronológico de bordaje y tratamiento inicial

\begin{tabular}{l|l|l|l|l|}
$\begin{array}{l}\text { 12:00 h: síncope con } \\
\text { recuperación parcial del } \\
\text { estado de alerta }\end{array}$ & $\begin{array}{l}16: 00 \mathrm{~h} \text { : nuevo síncope } \\
\text { sin recuperación del } \\
\text { estado de despierto }\end{array}$ & $\begin{array}{l}\text { 17:31 h: valoración por } \\
\text { triaje e ingreso a sala de } \\
\text { estabilización }\end{array}$ & $\begin{array}{l}\text { 17:35 h: manejo } \\
\text { avanzado de la vía }\end{array}$ & $\begin{array}{l}17: 38 \mathrm{~h} \text { : toma de ECG } \\
\text { aérea }\end{array}$ \\
$\begin{array}{l}\text { 17:40 hasometría de } \\
\text { ingreso }\end{array}$
\end{tabular}

- Choque cardiogénico.

- Lesión renal aguda.

- Encefalopatía metabólica.

- Acidosis metabólica de anión GAP elevado.

- Enfermedad cerebrovascular isquémica/hemorrágica.

- Bloqueos y bradiarritmias.

- Intoxicaciones agudas.

\section{Tratamiento}

El tratamiento inicial se basó en manejo avanzado de la vía aérea, reanimación hídrica a base de soluciones cristaloides y administración de medicamentos inotrópicos-cronotrópicos positivos intravenosos. Una vez que se obtuvo el ECG se integró el diagnóstico de IAM en cara inferior, con extensión eléctrica y hemodinámica al ventrículo derecho, y al estar en periodo de ventana para terapia de reperfusión coronaria farmacológica se administraron anticoagulantes y antiagregantes plaquetarios en dosis de carga y mantenimiento. La figura 3 muestra el electrocardiograma posterior a la administración de tenecteplasa. A los 40 minutos del contacto con el paciente se realizó trombólisis farmacológica con tenecteplasa, $35 \mathrm{mg}$ en bolo intravenoso, logrando criterios de reperfusión. De manera concomitante, el paciente presentó criterios gasométricos de CAD grave, por lo que se continuó la administración de líquidos intravenosos y se iniciaron la reposición electrolítica con $\mathrm{KCl}$ e insulina intravenosa por bomba de infusión. En la tabla 1 se resume el abordaje y tratamiento inicial del paciente.

\section{Desenlace y seguimiento}

El paciente requirió manejo avanzado de la vía aérea por deterioro neurológico, con datos clínicos de choque cardiogénico secundario a IAMCEST inferior con extensión mecánica y eléctrica al ventrículo
Tabla 2. Orden cronológico de los resultados bioquímicos

\begin{tabular}{|l|c|c|c|c|}
\hline Variable de laboratorio & Ingreso & Día 1 & Día 2 & Día 3 \\
\hline $\mathrm{pH}$ & 7.03 & 7.32 & 7.13 & 7.37 \\
\hline $\mathrm{pO}_{2}$ & 48 & 231 & 42 & 143 \\
\hline $\mathrm{pCO}_{2}$ & 59 & 38 & 44 & 34 \\
\hline $\mathrm{HCO}_{3}$ & 15.6 & 19.3 & 14.4 & 19.7 \\
\hline $\mathrm{BE}$ & -15.2 & -6.5 & -16.4 & -5.6 \\
\hline Lactato & 7.3 & 2.3 & 1.9 & 1.3 \\
\hline Hemoglobina & 13.3 & - & 10 & 10.3 \\
\hline Glucosa & 545 & - & 257 & 162 \\
\hline Creatinina & 1.6 & - & 3 & 3.4 \\
\hline CPK & - & 5261 & 2273 & 1139 \\
\hline CPK MB & 359 & 554 & 152 & 98 \\
\hline Sodio sérico & 139 & 144 & 137 & 140 \\
\hline Potasio sérico & 4.2 & 3.5 & 6.5 & 4.5 \\
\hline Cetonas en orina & $(-)$ & $(+)$ & - & - \\
\hline
\end{tabular}

BE: exceso de base; CPK: creatinfosfoquinasa.

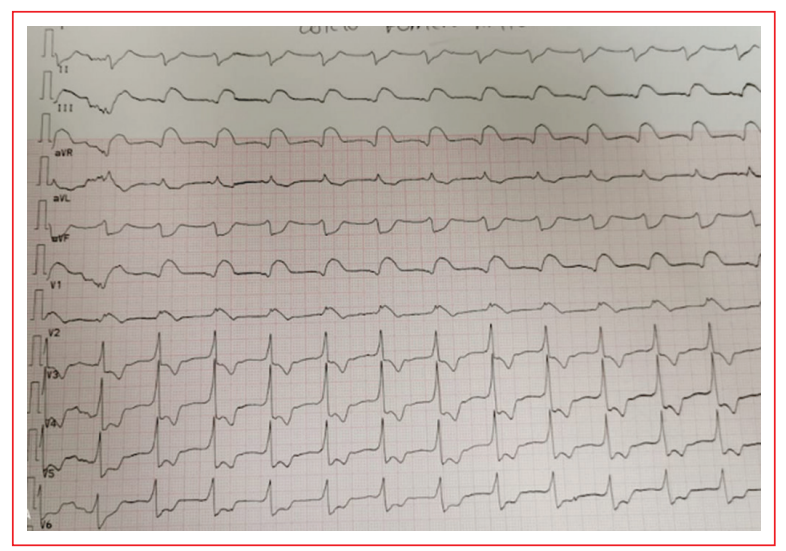

Figura 3. Electrocardiograma posterior a terapia de reperfusión farmacológica. 
Tabla 3. Línea de tiempo del abordaje terapéutico

\begin{tabular}{|c|c|c|c|c|c|}
\hline 29.10.21 & 30.10 .21 & 31.10.21 & 01.11 .21 & 02.11 .21 & 03.11 .21 \\
\hline $\begin{array}{l}\text { Ingreso a sala de } \\
\text { reanimación. Manejo de } \\
\text { vía aérea, colocación de } \\
\text { catéter central. } \\
\text { Trombólisis farmacológica. } \\
\text { Reposición } \\
\text { hidroelectrolítica e } \\
\text { insulina en infusión. Se } \\
\text { inicia vasopresor tipo } \\
\text { norepinefrina y dopamina. }\end{array}$ & $\begin{array}{l}\text { Cambio de } \\
\text { vasopresor a } \\
\text { dobutamina. } \\
\text { Continúa } \\
\text { aporte de } \\
\text { insulina por } \\
\text { infusión. } \\
\text { Disminución } \\
\text { de índice } \\
\text { urinario. Se } \\
\text { inicia } \\
\text { antiarrítmico } \\
\text { tipo } \\
\text { amiodarona. }\end{array}$ & $\begin{array}{l}\text { Disminución } \\
\text { gradual de } \\
\text { vasopresores. } \\
\text { Paciente con } \\
\text { oliguria y } \\
\text { aumento de } \\
\text { azoados. Se } \\
\text { inicia reto de } \\
\text { furosemida e } \\
\text { infusión, con } \\
\text { mejoría de } \\
\text { volúmenes } \\
\text { urinarios. }\end{array}$ & $\begin{array}{l}\text { Criterios de } \\
\text { resolución de } \\
\text { cetoacidosis } \\
\text { diabética. Continúa } \\
\text { con adecuado } \\
\text { índice urinario. } \\
\text { Disminución de } \\
\text { biomarcadores } \\
\text { cardiacos. Inicio de } \\
\text { protocolo de } \\
\text { extubación. }\end{array}$ & $\begin{array}{l}\text { Salida a BIPAP bien } \\
\text { tolerada. Con apoyo } \\
\text { vasopresor tipo } \\
\text { norepinefrina } 1 \mu \mathrm{g} / \\
\mathrm{kg} / \mathrm{h} \text {. Se suspende } \\
\text { dobutamina. Presenta } \\
\text { taquicardia } \\
\text { ventricular paroxística } \\
\text { y se ajusta } \\
\text { antiarrítmico. }\end{array}$ & $\begin{array}{l}\text { Ecografía } \\
\text { transtorácica } \\
\text { muestra FEVI 30, } \\
\text { TAPSE 15, } \\
\text { derrame } \\
\text { pericárdico, } \\
\text { hipocinesia } \\
\text { septal. } \\
\text { Disminución de } \\
\text { azoados y } \\
\text { balance hídrico } \\
\text { negativo. Se envía } \\
\text { a tercer nivel. }\end{array}$ \\
\hline
\end{tabular}

BPAP: bilevel positive airway; FEVI: fracción de eyección de ventrículo izquierdo; TAPSE: triscuspid anular plane septal excursion.

derecho que requirió tratamiento con aminas vasoactivas. Acude en periodo de ventana y con criterios para realizar reperfusión coronaria farmacológica con tenecteplasa, presentado arritmia de reperfusión. Con criterios de CAD grave, se iniciaron reposición hidroelectrolítica y bomba de insulina. Valorado y aceptado por la unidad de cuidados intensivos, se continuó en esta el tratamiento a base de soluciones cristaloides y aporte electrolítico, vasopresor tipo dobutamina, anticoagulantes, antiagregantes, estabilizador de membrana e insulina de acción rápida en bomba de infusión. Por presentar taquicardia ventricular paroxística se inició tratamiento antiarrítmico tipo amiodarona. El día primero de noviembre del año 2021, cumpliendo criterios de resolución de CAD, con mejoría del estado hemodinámico y disminución de azoados, se suspende la sedación para realizar protocolo de extubación. Al día siguiente se procede al destete de la ventilación mecánica a BIPAP, con adecuada tolerancia; presenta taquicardia ventricular, que remite de manera espontánea. Tose, deglute, con adecuada mecánica ventilatoria, radiografía de tórax sin consolidaciones ni derrame pleural. Se realiza referencia a tercer nivel, a la unidad de cardiología intervencionista, para realización de una intervención coronaria percutánea.

\section{Discusión}

El IAMCEST y la CAD, por sí solos, son afecciones que representan una amenaza vital y deben ser identificadas y atendidas de manera oportuna, lo cual implica el cumplimiento de las metas de diagnóstico y tratamiento instituidas de ámbito internacional.
En el presente caso, un varón de 54 años, acude a urgencias por síncope, sin recuperación del estado de alerta, con ECG de ingreso compatible con síndrome isquémico coronario agudo tipo IAMCEST inferior con extensión eléctrica y hemodinámica al ventrículo derecho con bloqueo auriculoventricular completo. Presenta franco deterioro neurológico como dato de bajo gasto cardiaco, por lo que se da manejo avanzado de la vía aérea y se inician vasopresores para compensación hemodinámica. Concomitante a esto, cumple criterios bioquímicos para CAD grave, seguramente detonada por el evento isquémico coronario, que en conjunto representan una mortalidad cercana al $85 \%$.

El paciente se presentó a urgencias con equivalente anginoso y por ECG se diagnosticó infarto inferior. Al no contar con una sala de hemodinamia y cumplir criterios, se administra terapia de reperfusión farmacológica con tenecteplasa, aunada a amina vasoactiva tipo dopamina buscando un efecto alfa y dopaminérgico. Posterior a reanimación hídrica inicial se modificó amina vasoactiva a dobutamina. En su estancia en la unidad de cuidados intensivos se resuelve la CAD con soluciones cristaloides, reposición hidroelectrolítica e insulina en infusión. Desarrolla arritmia que requiere tratamiento antiarrítmico. Presenta una evolución hemodinámica favorable, permitiendo la disminución paulatina de los vasopresores y la extubación sin aparentes complicaciones o secuelas. Se envía a tercer nivel para tratamiento específico por cardiología intervencionista. En la tabla 3 se resume la evolución clínica y tratamiento paciente.

\section{Perspectivas del paciente}

No fue posible determinar las perspectivas del paciente. 


\section{Agradecimientos}

Al doctor Javier Franco por su paciencia y orientación, a la doctora Alicia Bartolón por su dedicación y entusiasmo, y a la maestra Sofía Caballero por su apoyo incondicional.

\section{Financiamiento}

No se requirió financiamiento.

\section{Conflicto de intereses}

Los autores declaran no tener ningún conflicto de intereses.

\section{Responsabilidades éticas}

Protección de personas y animales. Los autores declaran que para esta investigación no se han realizado experimentos en seres humanos ni en animales.

Confidencialidad de los datos. Los autores declaran que han seguido los protocolos de su centro de trabajo sobre la publicación de datos de pacientes.
Derecho a la privacidad y consentimiento informado. Los autores han obtenido el consentimiento informado de los pacientes y/o sujetos referidos en el artículo. Este documento obra en poder del autor de correspondencia.

\section{Bibliografía}

1. Borrayo G, Pérez G, Martínez O, Almeida E, Ramírez E, Estrada J, et al. Protocolo para atención de infarto agudo de miocardio en urgencias: código infarto. Rev Med Inst Mex Seguro Soc. 2017;55:233-46.

2. Thygesen K, Alpert J, Jaffe A, Chaitman B, Max J, Morrow D, et al. Fourth universal definition of myocardial infarction. Eur Heart J. 2018;40:237-69.

3. Borrayo G, Rosas M, Pérez G, Ramírez E, Almeida E, Arriaga J. Infarto agudo del miocardio con elevación del segmento ST: código I. Rev Med Inst Mex Seguro Soc. 2018;56:26-37.

4. Martínez M, coordinador. Infarto agudo de miocardio. México: Intersistemas; 2014.

5. Jiménez M, Arguedas C, Romero L. Coronary syndrome and other diagnosis result in under reporting of acute myocardial infarction in the Mexico Hospital, Costa Rica. Acta Médica Costarricense. 2013:55:24-30.

6. Jerjes C, Martínez C, Borrayo G, Borrayo J, Carrillo J, Juárez U, et al. Third national registry of acute coronary syndromes (RENASICA III). Arch Cardiol Mex. 2015;85:207-14.

7. Ibáñez B, James S, Agewall S, Antunes M, Bucciarelli C, Bueno H, et al. ESC Guidelines for the management of acute myocardial infarction in patients presenting with ST-segment elevation. Eur Heart J. 2018;39:119-77.

8. Gong F, Vaitenas I, Malisrie C, Maganti K. Mechanical complications of acute myocardial infarction. JAMA Cardiol. 2021;6:341-9.

9. Martínez C, Briseño J. Choque cardiogénico. En: Martínez C, Briseño J. SICA. Síndromes isquémicos coronarios agudos. 3. ${ }^{a}$ ed. México: Intersistemas; 2021. p. 109-28.

10. Kadel A, Thakur K, Rauniyar B, Adhikari R, Limbu D, Malla R. Acute myocardial infarction and diabetic ketoacidosis: the lethal duo. J Nepal Health Res Counc. 2021;19:212-4. 